\title{
A catastrophic cervical necrotizing fasciitis after tooth extraction
}

\author{
Wei-Lin Lin $^{1}$ - Ta-Chuan Yeh $^{2}$ Chueng-He Lu' ${ }^{1}$ Hsu-Kai Huang ${ }^{3}$. \\ Wen-Yi Chiu ${ }^{4,5}$
}

Received: 8 December 2015/Accepted: 12 December 2015/Published online: 28 December 2015 (C) SIMI 2015

This is a 56-year-old male patient who had a past history of type 2 diabetes with irregular control by medication for more than 10 years. He had the habits of cigarette smoking and betel nut chewing. He had a decayed tooth extraction by his dentist. One day later, the patient had severe pain and local swelling over the left cheek, and was dyspneic. Initially, he went to a regional hospital where esophagoscopy showed an erosive oropharynx. Subsequently, he was sent to our emergency department. On examination, erythema with a central eschar, marked swelling and local subcutaneous crepitation of neck were noted (Fig. 1a). The body temperature was $37.5^{\circ} \mathrm{C}$, and there was an increased respiration rate. Urgent non-contrast enhanced computed tomography (CT) scanning showed a pneumomediastinum with air tracked downward from the pharynx to the paraaortic space (Fig. 1b), inflammatory changes, pleural effusion, bilateral empyema, and a left side pneumothorax (Fig. 1c). The patient was developed impending respiratory

\section{Wen-Yi Chiu}

hanrogyi@gmail.com

1 Department of Anesthesiology, Tri-Service General Hospital, School of Medicine, National Defense Medical Center, Taipei, Taiwan, ROC

2 Department of Psychiatry, Tri-Service General Hospital, School of Medicine, National Defense Medical Center, Taipei, Taiwan, ROC

3 Division of Thoracic Surgery, Department of Surgery, TriService General Hospital, School of Medicine, National Defense Medical Center, Taipei, Taiwan, ROC

4 Department of Internal Medicine, Kaohsiung Armed Forces General Hospital, Kaohsiung, Taiwan, ROC

5 Department of Family Medicine, Zouying Branch of Kaohsiung Armed Forces General Hospital, No. 553, Junxiao Rd., Zuoying Dist., Kaohsiung 813, Taiwan, ROC failure. We performed tracheal intubation assisted with a video-optical intubation stylet system due to the inflamed airway, and no chance of performing a surgical airway.

An emergent surgery, a large area of debridement and drainage of the neck and mediastinum were performed by the surgeon. Necrotic soft tissue with a foul odor was removed during operation. The patient was admitted to the intensive care unit. The culture of the neck pus grew out Prevotella spp. The blood cultures showed two strains of bacteria: Serratia marcescens and Stenotrophomonas maltophilia. A broad-spectrum antibiotic with imipenem and cilastatin was administered.

During admission, debridement was repeated several times. It was a complicated decayed tooth extraction. A video-assisted thoracic decortication was performed. The wound over the neck did not heal despite the surgical intervention and dressing changes. The sepsis worsened followed by multiple organ failure with acute respiratory distress syndrome. After 3 weeks of treatment, he erupted a massive blood loss from the neck wound, and expired despite resuscitation efforts.

Cervical necrotizing fasciitis is a life-threatening illness, and is more likely with a preexisting diabetes mellitus, chronic alcoholism, intravenous drug abuse, immunocompromised status, and obesity. We report this case of cervical necrotizing fasciitis with deep neck space infection (DNSI) and subsequent descending necrotizing mediastinitis. Fatal complications ensued after a dental extraction. Diagnostic criteria for descending necrotizing mediastinitis are suggested by Estrera et al. [1], They are: (1) a clinical manifestation of severe oropharyngeal infection, (2) the radiologic features of mediastinitis on CT, (3) documentation of a necrotizing mediastinal infection at surgery or on postmortem examination, and (4) an established relationship between oropharyngeal infection and development 


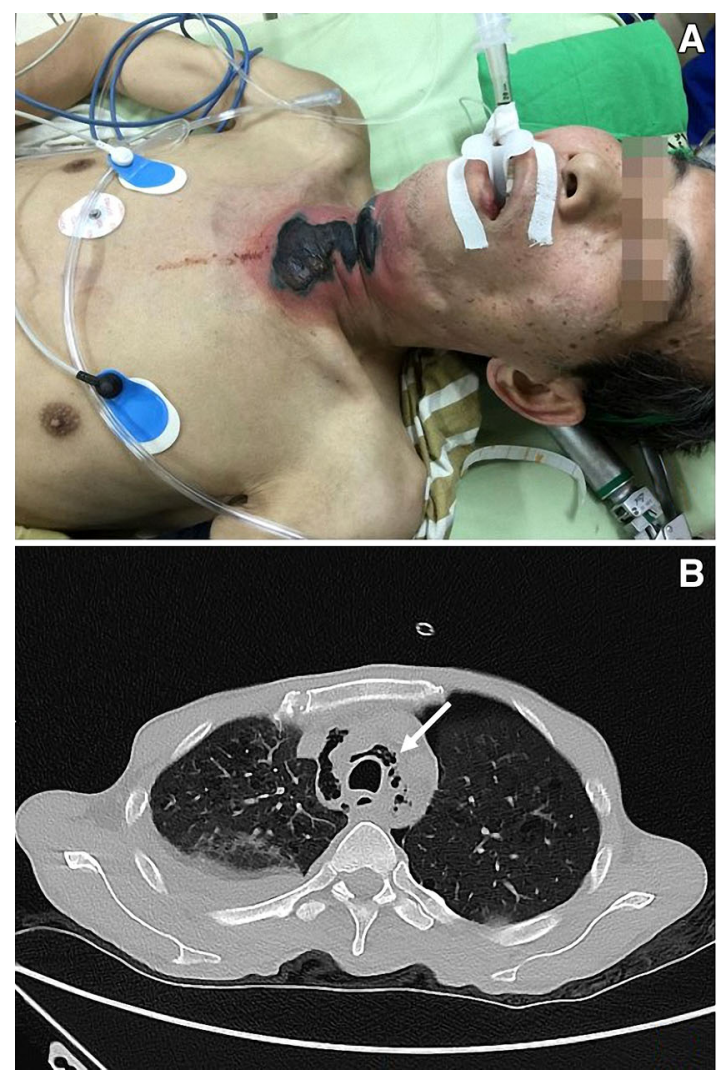

Fig. 1 a Erythema with a central eschar of neck. b CT scan at the level of the subaortic window showing abnormal gas collections of mediastinum (arrow) and a pleural effusion. c CT scan with sagittal

of descending necrotizing mediastinitis. When descending mediastinitis occurs, the reported mortality rate is $25-40 \%$ [2].

Overall, dental infection (43\%) remains the most common cause of cervical necrotizing fasciitis, but malnutrition, poor dental hygiene, smoking, and tobacco and beetle nut chewing may also increase the prevalence rate of odontogenic DNSI [3, 4]. For effective management, early diagnosis is important. Optimal surgical drainage of purulent abscesses, close monitoring, and broad-spectrum antibiotics therapy can significantly reduce the mortality rate [2]. In our patient, delay in seeking timely medical care and poor underlying condition led to catastrophic consequences.

\section{Compliance with ethical standards}

Conflict of interest The authors declare that they have no conflict of interest.

Statement of human and animal rights We declare the research documented in the present manuscript involving human participants

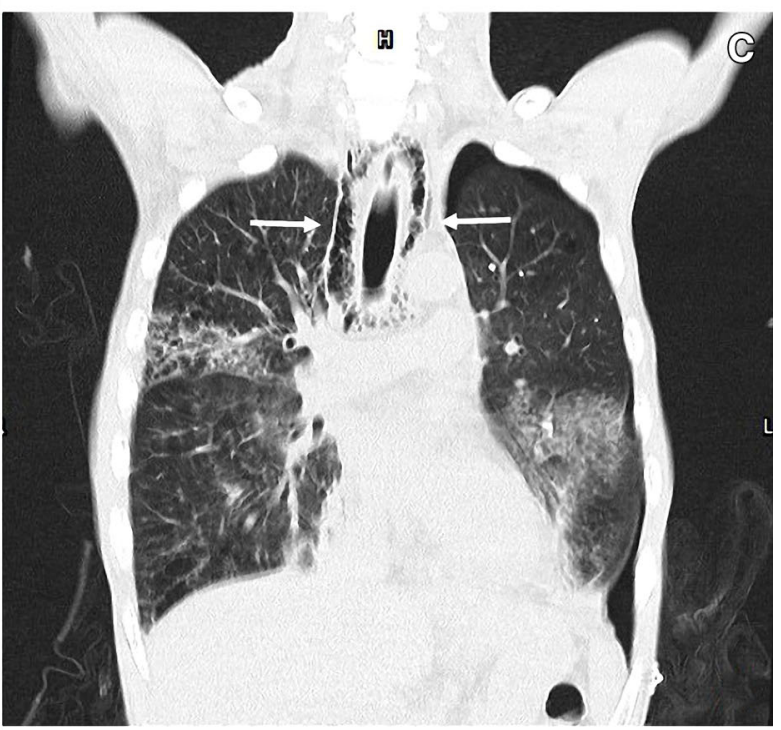

reconstruction showing pneumomediastinum with air dissection (arrow), bilateral empyema, and a left-sided pneumothorax

were in accordance with the ethical standards of the institutional and/ or national research committee and with the 1964 Helsinki declaration and its later amendments or comparable ethical standards.

Informed consent Written informed consent was obtained from the patient.

\section{References}

1. Estrera AS, Landay MJ, Grisham JM et al (1983) Descending necrotizing mediastinitis. Surg Gynecol Obstet 157(6):545-552

2. Singhal P, Kejriwal N, Lin Z et al (2008) Optimal surgical management of descending necrotising mediastinitis: our experience and review of literature. Heart Lung Circ 17(2):124-128

3. Parhiscar A, Har-El G (2001) Deep neck abscess: a retrospective review of 210 cases. Ann Otol Rhinol Laryngol 110(11):1051-1054

4. Kataria G, Saxena A, Bhagat S et al (2015) Prevalence of odontogenic deep neck space infections (DNSI): a retrospective analysis of 76 cases of DNSI. Int J Otorhinolaryngol Head Neck Surg 1(1):11-16 